\title{
T follicular helper expansion and humoral-mediated rejection are independent of the HVEM/BTLA pathway
}

\author{
Jose-Ignacio Rodriguez-Barbosa ${ }^{1}$, Carlos Fernandez-Renedo ${ }^{1}$, Ana María Bravo Moral ${ }^{2}$, Leo Bühler ${ }^{3}$ \\ and Maria-Luisa del Rio ${ }^{1}$
}

The molecular pathways contributing to humoral-mediated allograft rejection are poorly defined. In this study, we assessed the role of the herpesvirus entry mediator/B- and T-lymphocyte attenuator (HVEM/BTLA) signalling pathway in the context of antibody-mediated allograft rejection. An experimental setting was designed to elucidate whether the blockade of HVEM/BTLA interactions could modulate de novo induction of host antidonor-specific antibodies during the course of graft rejection. To test this hypothesis, fully allogeneic major histocompatibility complex-mismatched skin grafts were transplanted onto the right flank of recipient mice that were treated with isotype control, anti-CD40L or modulatory antibodies of the HVEM/BTLA signalling pathway. The frequencies of CD4 T follicular helper (Tfh) cells (B220-, CD4+ CXCR5 + PD-1high), extrafollicular helper cells (B220-, CD4+ CXCR5 - PD-1+ and PD-1-) and germinal centre (GC) B cells (B220+Fas + GL7+) were analysed by flow cytometry in draining and non-draining lymph nodes at day 10 post transplantation during the acute phase of graft rejection. The host antidonor isotype-specific humoral immune response was also assessed. Whereas blockade of the CD40/CD40L pathway was highly effective in preventing the allogeneic humoral immune response, antibody-mediated blockade of the HVEM/BTLA-interacting pathway affected neither the expansion of Tfh cells nor the expansion of GC B cells. Consequently, the course of the host antidonor antibody-mediated response proceeded normally, without detectable evidence of impaired development. In summary, these data indicate that HVEM/BTLA interactions are dispensable for the formation of de novo host antidonor isotype-specific antibodies in transplantation.

Cellular \& Molecular Immunology (2017) 14, 497-510; doi:10.1038/cmi.2015.101; published online 29 February 2016

Keywords: BTLA (CD272); humoral immunity; HVEM (TNFRSF14, CD270); T follicular helper cells;

transplantation

\section{INTRODUCTION}

Organ transplantation is a medical intervention that is used to prolong the lifespan of patients suffering from end-stage terminal illnesses. Despite the potent immunosuppression treatments that are currently available for the prevention and treatment of graft rejection, a large proportion of solid-organ allografts (approximately 50\%) are lost 10 years after transplantation. ${ }^{1}$ Antibody-mediated rejection (ABMR) is one of the leading causes of chronic graft rejection, which is refractory to current maintenance protocols of immunosuppression in many instances. ${ }^{2-4}$ Therefore, to improve the ways in which we treat ABMR, a better depth of knowledge regarding the molecular pathways that contribute to the process of T-cell to B-cell help would permit a more precise and specific manner to tackle this clinical challenge through biologics rather than conventional immunosuppression.

The production of de novo antibodies against donor major histocompatibility complex (MHC)-mismatched antigens after transplantation depends on the cognate interaction between $\mathrm{CD} 4 \mathrm{~T}$ follicular helper cells (Tfh) that are primed by donor alloantigens presented in the context of host APCs (the indirect pathway of antigen presentation) and B lymphocytes that recognize soluble and membrane-bound alloantigens. ${ }^{5-7}$ The requirement of T-cell help for B-cell antibody production was first described in the late sixties. ${ }^{8,9} \mathrm{CD} 4 \mathrm{Tfh}$ cells of the secondary lymphoid organs are now recognized as the immune cells that are responsible for orchestrating the molecular interactions that provide help to B cells. This help is promoted through soluble

\footnotetext{
${ }^{1}$ Transplantation Immunobiology Section, Institute of Biomedicine, University of Leon and Castilla and Leon Regional Transplantation Coordination, Leon University Hospital, Leon, Spain; ${ }^{2}$ Department of Veterinary Clinical Sciences, University of Santiago de Compostela, Veterinary Faculty, Lugo, Spain and ${ }^{3}$ Visceral and Transplantation Surgery, Department of Surgery, University Hospitals of Geneva and Faculty of Medicine, Geneva, Switzerland Correspondence: ${ }^{1} \mathrm{~J}-\mathrm{I}$ Rodríguez-Barbosa (E-mail: ignacio.barbosa@unileon.es) or M-L del Rio (E-mail: mlriogon@saludcastillayleon.es) Received: 19 July 2015; Revised: 2 November 2015; Accepted: 14 November 2015
} 
factors (e.g. IL-21, IL-4, and IL-6) and cell-bound molecules, such as ICOS (inducible T-cell co-stimulator) and CD40L that deliver costimulatory signals to ICOS-L and CD40 expressing alloantigen-specific B cells. ${ }^{10}$ These interactions drive B-cell differentiation towards extrafollicular short-lived plasma blasts and intrafollicular long-lived plasma cells, which produce antibodies, as well as the formation of memory B cells. ${ }^{10-13}$ The transcriptional repressor B-cell lymphoma 6 seems to regulate the genetic program of CD4Tfh cell differentiation and is a suitable biomarker that is useful in distinguishing these cells from other CD4 T cells (e.g. Th1, Th2, Th17, and T-regulatory). ${ }^{14}$

Somatic hypermutation and selection of B cells that express immunoglobulin variants with improved antigen affinity, along with the process of immunoglobulin class switching that occurs in allo-specific activated $\mathrm{B}$ cells requires cognate CD4 $\mathrm{T}$ help during the germinal centre (GC) reaction. ${ }^{15,16}$ Upon antigenic stimulation, CD4 T cells upregulate CXCR5 and first migrate to the interfollicular area that is in close proximity to the T-cell/ B-cell border through attraction to the follicle stromal cell-derived CXCL13 chemokine. CD4 T cells then further migrate to the follicle to prime follicular B cells and form the GC reaction. ${ }^{17}$

Herpesvirus entry mediator (HVEM) is a member of the tumour necrosis factor receptor superfamily (TNFRSF14, $\mathrm{CD} 270$ ) and represents the unique known molecular partner of B- and T-lymphocyte attenuator (BTLA, CD272), a member of the immunoglobulin superfamily. ${ }^{18}$ Whereas HVEM exhibits a broad pattern of expression, which extends to haematopoietic and non-haematopoietic cells, BTLA expression is restricted to the haematopoietic compartment, with higher expression on B cells than on T cells. ${ }^{19-21}$ Under resting conditions, HVEM expressed on T cells interacts with BTLA on the same cell (cis-interaction) controlling the functional status of the $T$ cell and thus impeding the establishment of trans HVEM or BTLA interactions. However, upon T-cell activation, the kinetics of the BTLA and HVEM expression levels change overtime and BTLA augments its expression, whereas HVEM expression diminishes; therefore, allowing trans interactions to occur between $\mathrm{T}$ and $\mathrm{B}$ cells, as well as between other cell types. $^{20,22}$ The experimental evidence of the role of the BTLA/ HVEM pathway in humoral immunity comes from the analysis of the phenotype of BTLA-deficient mice, which revealed defects in the regulation of this branch of immunity with implications in the development of autoimmune diseases. ${ }^{23-25}$ As the HVEM receptor is the unique known partner of BTLA and both molecules are expressed on T and B cells, we postulated that the HVEM/BTLA signalling pathway may participate in providing CD4 help to B cells for the production of donorspecific antibodies. ${ }^{20}$ With this notion in mind, we set up an experimental approach to elucidate the role of this interaction in the context of the humoral immune response during the acute phase of graft rejection.

\section{MATERIALS AND METHODS}

\section{Animals}

Eight- to 12-week-old female C57BL/6 (B6, H-2 ${ }^{\mathrm{b}}$ ) and BALB/c $\left(\mathrm{H}-2^{\mathrm{d}}\right)$ mice were bred at the University of Leon (Spain) animal facility. All rodent experiments were performed in accordance with the European and National Guidelines for Animal Care of Laboratory Animals, following an animal protocol that was approved by both the Local Ethical Committee for Animal Research (School of Veterinary Medicine, University of Leon) and the Regional Assessor Committee for Animal Welfare.

\section{Skin graft transplantation model}

C57BL/6 and BALB/c skin graft transplantation surgery was carried out as previously described. ${ }^{26} \mathrm{C} 57 \mathrm{BL} / 6$ and BALB/C recipients were anesthetized and grafted with allogeneic tail skin on the right flank. The grafts were covered with Vaseline gauze, and bandages were removed at day 7 after transplantation. The right draining lymph nodes (LNs) and left nondraining axillar and brachial LNs were collected at days 10 and 20 post transplantation.

\section{Antibodies for in vivo use and flow cytometry}

Hybridoma cell lines that secreted an anti-HVEM monoclonal antibody (mAb) (clone 6C9, blocking antibody of the HVEM/ BTLA interaction), ${ }^{20}$ anti-BTLA mAb (clone 4G12b, downmodulates BTLA receptor expression), ${ }^{21,27}$ anti-CD40L mAb (clone MR1, blocking/depleting antibody) $)^{28-30}$ or an isotype-matched control rat $\operatorname{IgG}_{2 \mathrm{a}}$ (antiplant cytokinin, clone AFRC-MAC-157) antibody were grown in Serum free medium (SFM) medium Gibco (Grand Island, NY 14072, USA) supplemented with IgG-depleted foetal calf serum (less than 1\%) in spinner flasks. Cell culture supernatants were pre-filtered and then loaded onto a protein G-Sepharose column, and affinity chromatography was performed for the purification of the immunoglobulins. The eluted fraction was neutralized and dialysed against Phosphate Buffered Saline (PBS), passed through a $0.45-\mu \mathrm{m}$ filter and stored frozen at a concentration of $1 \mathrm{mg} / \mathrm{ml}$. These antibodies were thus prepared for in vivo use. The first 1-mg dose of each mAb was injected intraperitoneally at the time of transplantation, and a second dose was administered at day 10 post transplantation, according to the experimental set up.

The following fluorochrome-labelled antibodies were used: B220 (RA3-6B2), CD4 (GK1.5), CXCR5 (L138D7), PD-1 (29F.1A12), T- and B-cell activation antigen (GL7), CD95 (15A7) and ICOS (C398.4A). Flow antibodies were purchased from Biolegend (London, United Kingdom), eBioscience (Hatfield, Ireland, United Kingdom), Beckton Dickinson (Heidelberg, Germany), except for the anti-HVEM (clone 5A8) and anti-BTLA mAbs (clone 4G12b), which were purified and biotinylated in our laboratory. ${ }^{20,21}$ Cell suspensions were incubated with $2 \mu \mathrm{g} / \mathrm{ml}$ of blocking anti-Fc $\gamma \mathrm{R} \mathrm{mAb}$ (clone 2.4G2, rat $\mathrm{IgG}_{2 \mathrm{~b}}$ anti-Fc $\gamma$ RII/III $\mathrm{mAb}$ ) before staining with the fluorochrome-labelled antibodies to reduce nonspecific binding. ${ }^{31}$ Dead cells and debris were excluded from the acquisition gate as positive events for propidium iodide staining. Samples were acquired on a Cyan 9 cytometer (Beckman Coulter, Miami, FL, USA), and the data analysis was performed using WinList version 8.0 (Verity Software House, Topsham, ME, USA). 
The serum levels of anti-BTLA, anti-HVEM and isotype control mAbs (all rat IgG2a) and the clearance kinetics were determined in recipient naïve mice that were intraperitoneally administered with $1 \mathrm{mg}$ of each antibody at a dose equivalent to $40 \mathrm{mg} / \mathrm{kg}$ of body weight. Serum was collected from blood that was obtained on days 1, 6 and 10 post injection. The rat IgG2a levels were quantified in the serum samples by sandwich ELISA using a standard curve of known concentrations of commercial rat $\operatorname{IgG}_{2 \mathrm{a}}$ (clone R35-95). The binding of the injected antibody to BTLA and HVEM that were expressed on the cell surface of B cells and T cells was also monitored in treated mice to ensure saturation of the receptors. The half-life of antiCD40L antibody (clone MR1, hamster IgG) has been reported by several groups who intraperitoneally injected antibody doses ranging from 0.5 to $2 \mathrm{mg}$ at the time of transplantation, in which the half-life of anti-CD40L antibody was estimated at 10 days. ${ }^{32,33}$ The half-lives of the antibodies targeting the HVEM/BTLA pathway, both antibodies that were bound to the receptors and the circulating unbound antibody, were calculated to ensure that the surface receptor was saturated over time and an excess amount of antibody was present in the serum of the treated mice using the following exponential decay formulas: $\mathrm{Nt}=\mathrm{N} 0(1 / 2)^{t / t 1 / 2}, \mathrm{Nt}=\mathrm{N} 0 \mathrm{e}^{-\mathrm{t} / \tau}, \mathrm{Nt}=\mathrm{N} 0 \mathrm{e}^{-\lambda t}$, where $\mathrm{N} 0$ indicates the initial quantity present in the serum 1 day after the administration of the therapeutic antibody, $\mathrm{Nt}$ indicates the quantity that still remained after a time $t, t_{1 / 2}$ indicates the half-life, $\tau$ indicates the mean lifetime, and $\lambda$ indicates the decay constant.

\section{Host antidonor-specific antibody response}

Biotinylated $\mathrm{H}-2 \mathrm{~K}^{\mathrm{b}}$ and biotinylated $\mathrm{H}-2 \mathrm{~K}^{\mathrm{d}}$ monomers were synthesized at the National Institutes of Health tetramer core facility (Atlanta, GA, USA) and were used in this study under a collaborative agreement. Two different approaches were adopted for the measurement of the host antidonor humoral immune response. Serum samples were collected from the different experimental groups at days 10 and 20 after transplantation. Then, they were diluted (at 1:20) and incubated for 1 hour with $0.5 \times 10^{6}$ donor thymocytes. The extent of the host antidonor isotype-specific humoral immune response was determined evaluating the mean fluorescence intensity values of the staining. Additionally, we also took advantage of an ELISA in which $1 \mu \mathrm{g} / \mathrm{ml}$ of biotinylated $\mathrm{H}-2 \mathrm{~K}^{\mathrm{d}}$ monomers and $1 \mu \mathrm{g} / \mathrm{ml}$ of biotinylated $\mathrm{H}-2 \mathrm{~K}^{\mathrm{b}}$ monomers were bound to streptavidin-coated plates $(1 \mu \mathrm{g} / \mathrm{ml}$ streptavidin, Thermofisher (Waltham, MA USA)). Then, the plates were blocked with PBS containing $1 \% 9$. Bovine Serum Albumin (BSA) for 1 hour at room temperature and washed; then, 1:20 diluted serum samples were added to the coated plates and further incubated for 2 hours at room temperature. The plates were washed three times with PBS containing $0.05 \%$ Tween 20 and then further incubated with horseradish peroxidase-labelled antimouse IgG Promega (Madison, WI, USA). The reaction was finally developed by adding $3,3^{\prime}, 5,5^{\prime}-$ tetramethylbenzidine substrate, according to the manufacturer's instructions Merck (Kenilworth, NJ, USA). The reaction was stopped with $2 \mathrm{M}$ sulphuric acid, and the absorbance was measured at a wavelength of $450 \mathrm{~nm}$ using a multi-well spectrophotometer plate reader BioRad (California, USA).

\section{Statistical analysis}

The data were organized in Excel worksheets and analysed using GraphPad prism Version 5. The parametric Student's $t$ test was used for the statistical comparison of the means between the non-draining and draining LNs in each experimental group. One-way analysis of variance test and a post analysis based on Tukey's test were applied to compare the differences of the means among either non-draining or draining LN groups. These statistical studies were performed under independent data conditions, in which a normality test (Kolmorogov test) and equal variances among the groups were considered (Bartlett's test). Skin graft survival was calculated using the Kaplan-Meier life table method, and statistical analysis for the comparison of the survival curves was performed with the log rank (Mantel-Cox) test. Statistical analysis was performed using GraphPad Prism 5.0 software, and a $P$-value of less than 0.05 was considered statistically significant.

\section{RESULTS}

The HVEM/BTLA signalling pathway is not required for the expansion of alloreactive $\mathrm{CD} 4 \mathrm{Tfh}\left(\mathrm{CXCR}^{+}\right)$cells and extrafollicular CD4 $\mathrm{T}$ cells $\left(\mathrm{CXCR}^{-}\right)$

To evaluate the role of the HVEM/BTLA signalling pathway in the clonal expansion of Tfh cells, BALB/c tail skin was grafted on the right flank of recipient C57BL/6 mice that were treated with an isotype-matched control $\mathrm{mAb}$, an anti-CD40L $\mathrm{mAb}$ or mAbs against BTLA/HVEM pathway molecules. ${ }^{20,21}$ Nontransplanted naïve mice and syngeneic skin grafts were added to the experimental set up to monitor the influence of the inflammatory response due to the surgery. To ensure efficient surface receptor capping by the therapeutic antibodies, the recipient mice were intraperitoneally injected with $1 \mathrm{mg}$ of antibody and the binding of the antibodies to BTLA and HVEM was monitored on lymphoid cells for up to 10 days. Furthermore, the half-lives of the antibodies that were administered in vivo to recipient mice were monitored: the half-life for the anti-BTLA antibody was 4.73 days and the anti-HVEM antibody half-life was 8.39 days (Supplementary Table S1 and Supplementary Figure S1).

Ten days after transplantation, during the acute phase of the rejection, the recipient mice were euthanized and the right draining and left non-draining brachial and axillar LNs were collected and dissociated and the absolute number of LN cells was calculated. In response to alloantigen stimulation, the draining LNs had significantly increased cell numbers compared with the non-draining LNs in all of the experimental groups; however, this was observed to a much lower extent than in the anti-CD40L-treated group (Figure 1a, left panel). This result confirmed the well-described potent functional activity of anti-CD40L mAb clone MR1) treatment in preventing LN swelling and also proves that CD4 T cells help to promote GC reactions. ${ }^{34}$ The anti-BTLA and anti-HVEM mAb treatments, however, did not alter the cell numbers in the draining LNs 

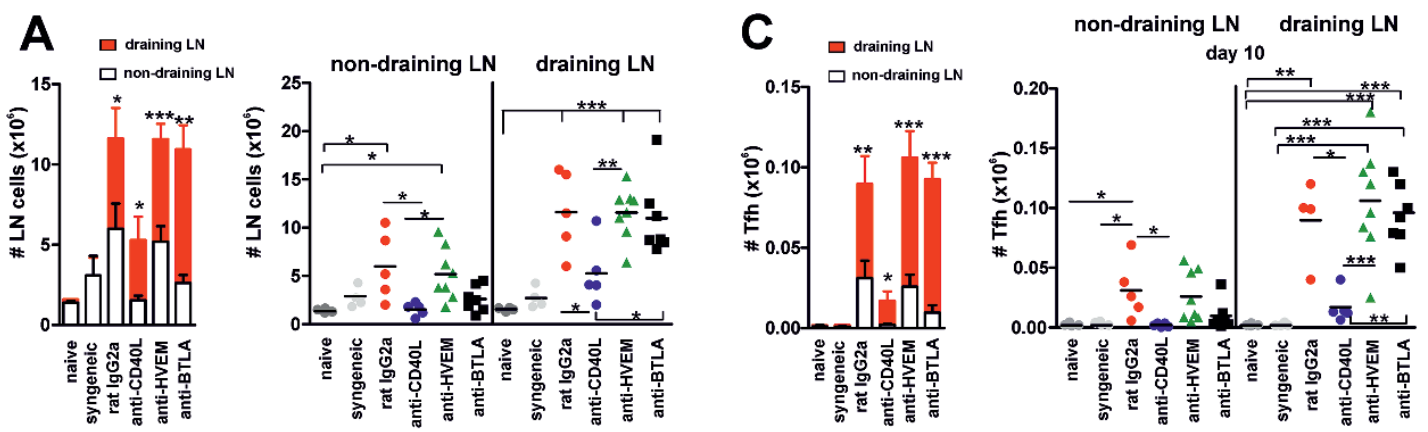

B non-draining LN
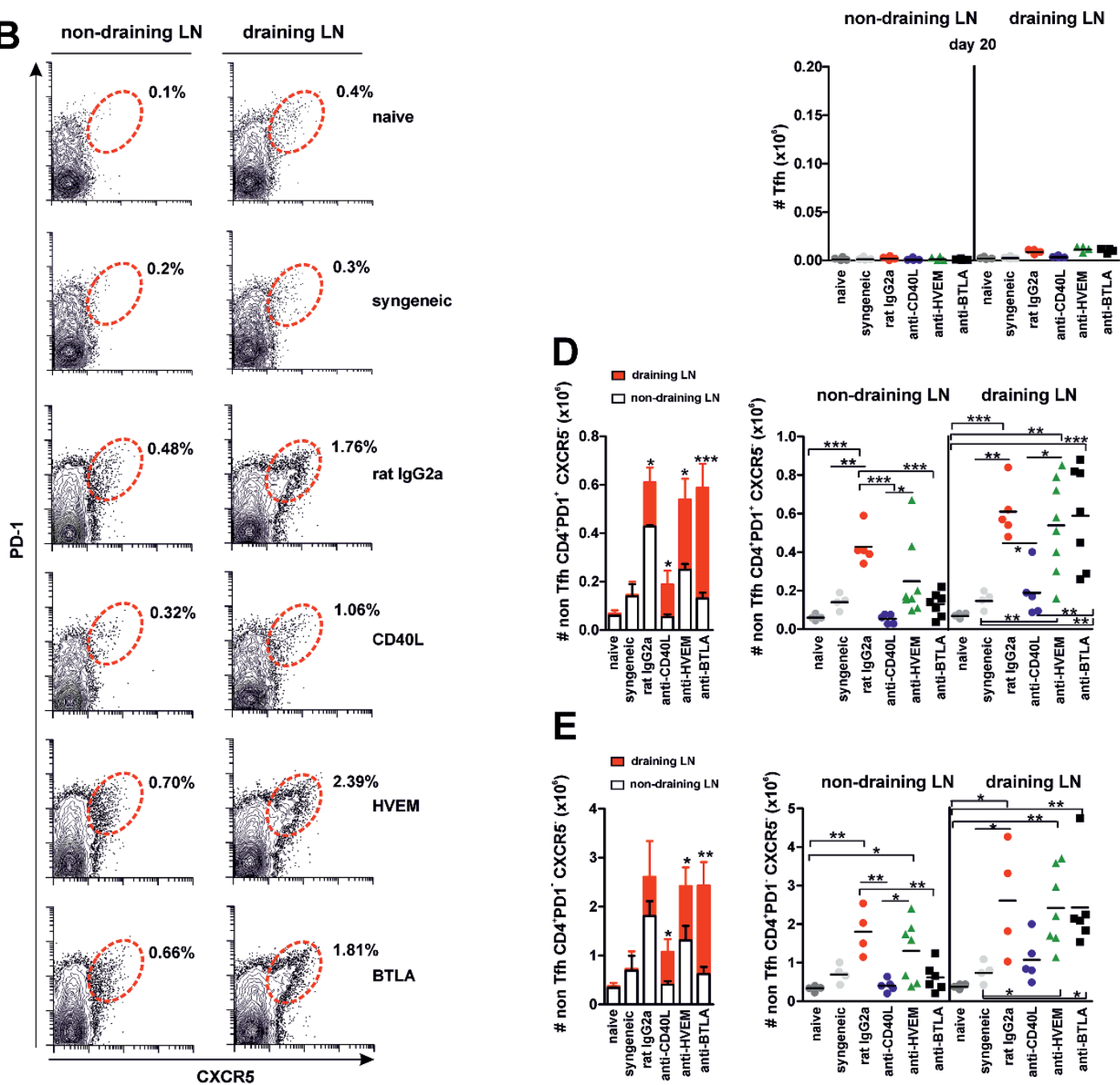

Figure 1 The expansion of Tfh $\mathrm{CXCR} 5^{+}$and extrafollicular helper $\mathrm{CXCR} 5^{-}$cells is independent of the HVEM/BTLA signalling pathway. The absolute cell numbers in the non-draining and draining LNs were plotted in superimposed graphs and the existence of significant differences was evaluated for each experimental group (a, left panel). The absolute cell numbers in the draining and non-draining LNs of the different experimental groups are displayed 10 days after transplantation of BALB/c skin onto the right flank of C57BL/6 recipient mice (a, right panel). (b) CD4 Tfh and extrafollicular T helper cells were phenotypically characterized by flow cytometry as B220- $\mathrm{CD}^{+} \mathrm{CXCR}^{+} \mathrm{PD}-1^{+}$(dotted red circle) and B220 ${ }^{-}$ $\mathrm{CD}^{+}{ }^{+}$CXCR5 ${ }^{-} \mathrm{PD}-1^{+}$and PD- ${ }^{-}$, respectively, in the non-draining and draining LNs. The same description as in Figure $1 \mathrm{a}$ applies for the Tfh cell numbers in the non-draining and draining LNs of each experimental group at days 10 (c, left and middle panel) and 20 after transplantation of

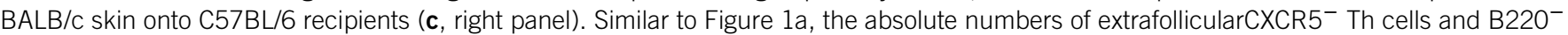
$\mathrm{CD}^{+}{ }^{+} \mathrm{PD} 1^{+}$and $\mathrm{PD} 1^{-}$cells are shown $(\mathbf{d}-\mathbf{e})$. The data are presented as the means $\pm \mathrm{SEM}$. The statistical significance and $P$-values were calculated using unpaired Student's $t$-test for the comparisons of means between the draining versus non-draining LNs. One-way analyses of variance were applied for the comparison of the means from different experimental groups with regard to the draining or non-draining LNs. The degree of significance was represented as $* P<0.05$; $* * P<0.005$; $* * P<0.0005$. All plots display a pool of data from three independent experiments. BTLA, B- and T-lymphocyte attenuator; HVEM, herpesvirus entry mediator; Tfh, T follicular helper cells. 
compared with the isotype-treated control (Figure 1a, right panel).

We next evaluated whether CD4 Tfh cell clonal expansion was affected in the B6 allogeneic skin graft recipient mice. The gating strategy removed the $\mathrm{B} 220^{+}$cells to analyse the Tfh $\mathrm{CXCR}^{+}{ }^{+} \mathrm{PD} 1^{+}$population within the gated $\mathrm{CD} 4^{+} \mathrm{T}$-cell population, which allowed for Tfh cell percentage determinations in the non-draining and draining LNs (Figure 1b).

The absolute number of CD4 Tfh cells were significantly augmented in the draining LNs compared with the non-draining LNs in response to the alloantigen stimulation in all the experimental groups at day 10 after transplantation (Figure 1c, left and middle panel), and the Tfh response contracted by day 20 in all B6 mouse groups that received allogeneic BALB/c skin grafts (Figure 1c, right panel). The treatment with antibodies against HVEM/BTLA pathway molecules did not affect the Tfh cell numbers in the draining LNs compared with those observed in the isotype-matched treated control (Figure 1c, right panel).

The expansion of the non-Tfh populations, including the CXCR5 ${ }^{-} \mathrm{PD}-1^{+}$cells (Figure 1d) and $\mathrm{CXCR} 5^{-} \mathrm{PD}-1^{-}$cells (Figure 1e), was also examined within the $\mathrm{B}_{2} 20^{-} \mathrm{CD} 4^{+}$population in the non-draining and draining LNs. Interestingly, the non-Tfh cell $\left(\mathrm{CXCR}^{-}\right)$analysis showed a statistically significant increase in PD- $1^{+}$(Figure $1 \mathrm{~d}$ ) and PD-1 ${ }^{-}$cell numbers (Figure 1e) in the draining compared with the non-draining LNs. This result supports the notion that the extrafollicularCD4 Thelper cell population also expanded in response to alloantigen stimulation and was probably involved in driving the differentiation of extrafollicular B cells to antibody-secreting short-lived plasmablasts.

The expansion of extrafollicular $\mathrm{CD} 4^{+} \mathrm{PD}-1^{+} \mathrm{T}$ cells was significantly abolished after the administration of a single dose of anti-CD40L mAb at the time of transplantation in the draining LNs compared with the rest of the experimental groups (Figure 1d, right panel). Regarding the extrafollicular $\mathrm{CD} 4^{+}$ $\mathrm{PD}-1^{-} \mathrm{T}$ cells, this subpopulation was also reduced after the anti-CD40L administration; however, it did not reach statistical significance (Figure 1e, right panel). This emphasizes the role of CD40/CD40L interactions in the expansion of extrafollicular Th cells, which in turn would provide help to activated B cells to drive their differentiation towards shortlived plasma blasts for the early production of de novo host antidonor-specific antibodies. Similar to the results obtained for the Tfh cells, the expansion of extrafollicular helper cells was not affected by treatment with antibodies against the HVEM/ BTLA signalling pathway molecules.

These data indicate that LN expansion of allogeneic follicular and extrafollicular $\mathrm{T}$ helper cells is an immunological event that occurs independent of HVEM/BTLA interactions during allograft rejection.

GC B-cell clonal expansion is independent of HVEM/BTLA interactions in allograft rejection

Given that CD4 Tfh cell expansion was not altered after HVEM/ BTLA blockade and because GC formation depends on cognate $\mathrm{T}$-cell help to $\mathrm{B}$ cells, we anticipated that $\mathrm{GC}$ reactions in $\mathrm{B} 6$ mice that are sensitized with fully MHC-mismatched skin allografts would also be unaffected. To gain insight into this immunological mechanism, we monitored the total B-cell numbers $\left(\mathrm{B} 220^{+}\right)$in non-draining and draining LNs. The B-cell expansion proceeded normally and to a much greater extent in the draining than in the non-draining LNs at day 10 after skin graft transplantation during the acute phase of rejection in all experimental groups except the non-transplanted naïve or syngeneic skin grafts control mice (Figure 2a, left panel). This result suggests that the stimulus of the allogeneic skin graft transplantation induced effective proliferation and accumulation of B cells in the draining LNs and consequently, the LNs increased in size. However, the increase in the B-cell numbers in the draining LNs was not significantly inhibited by the treatment with the mAbs that blocked the HVEM/BTLA pathway (Figure 2a, right panel).

Within the B-cell population, the major subpopulation in this compartment was the follicular B cells (PD- ${ }^{-}{ }^{-}$CXCR $5^{+}$ cells), which expanded to a substantially larger extent in the draining LNs than in the non-draining LNs (Figure 2b, left panel). Statistically significant differences were not found among the experimental groups draining LNs, except for the anti-CD40L-treated mice, in which follicular B-cell expansion was abolished due to the blockade of CD40L/CD40 interaction and the subsequent impairment of cognate $\mathrm{CD} 4 \mathrm{Tfh}$ cell help to $\mathrm{B}$ cells (Figure 2b, right panel).

Finally, and to gain information as to whether the HVEM/ BTLA interaction blockade could impact, to some extent, the formation of GCs during the acute phase of rejection, B220 ${ }^{+}$ cells expressing GC surface markers (Fas and GL7) were analysed at day 10 after transplantation in the non-draining and draining LNs as displayed in the schematic illustrated in Figure 2c. The absolute number of GC B cells significantly expanded in all experimental groups, except in the naïve mice, syngeneic controls and in anti-CD40L-treated mice, in which the GC reaction was practically absent in the draining and non-draining LNs (Figure 2d, left panel). The treatment with the HVEM/BTLA pathway antibodies did not affect the GC cell expansion level in the draining LNs as it was similar to the level observed in the mice receiving the isotype control antibody treatment at day 10 of transplantation (Figure 2d, middle panel). At day 20 post transplantation, the absolute number of GC B cells started to contract in parallel with the elimination of the allogeneic stimulus (Figure $2 \mathrm{~d}$, right panel).

The B cells, follicular B cells and GC B cells were unexpectedly reduced in the non-draining LNs in recipient mice that were treated with anti-BTLA, which suggested that BTLA expression was necessary for the survival of these B-cell populations in the resting state under non-inflammatory conditions (Figure 2a, right panel, Figure 2b, right panel and Figure 2d, middle panel).

Overall, the data confirm the notion that interfering with the HVEM/BTLA pathway does not impair GC cell expansion during the allogeneic immune response, although it may affect the survival of resting B cells. 
A
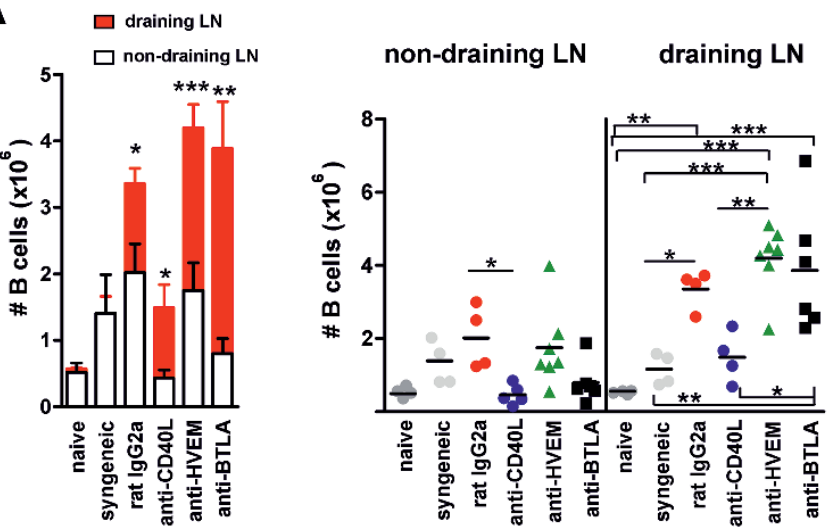

B
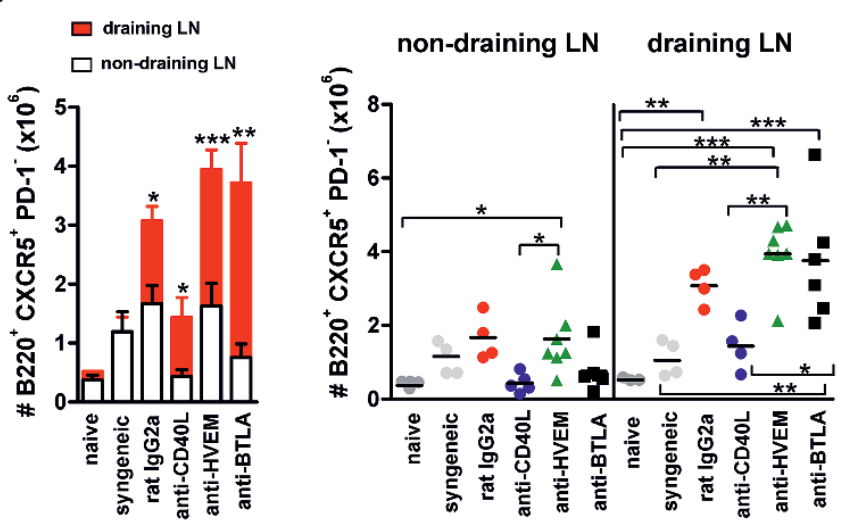

C

non-draining LN draining LN
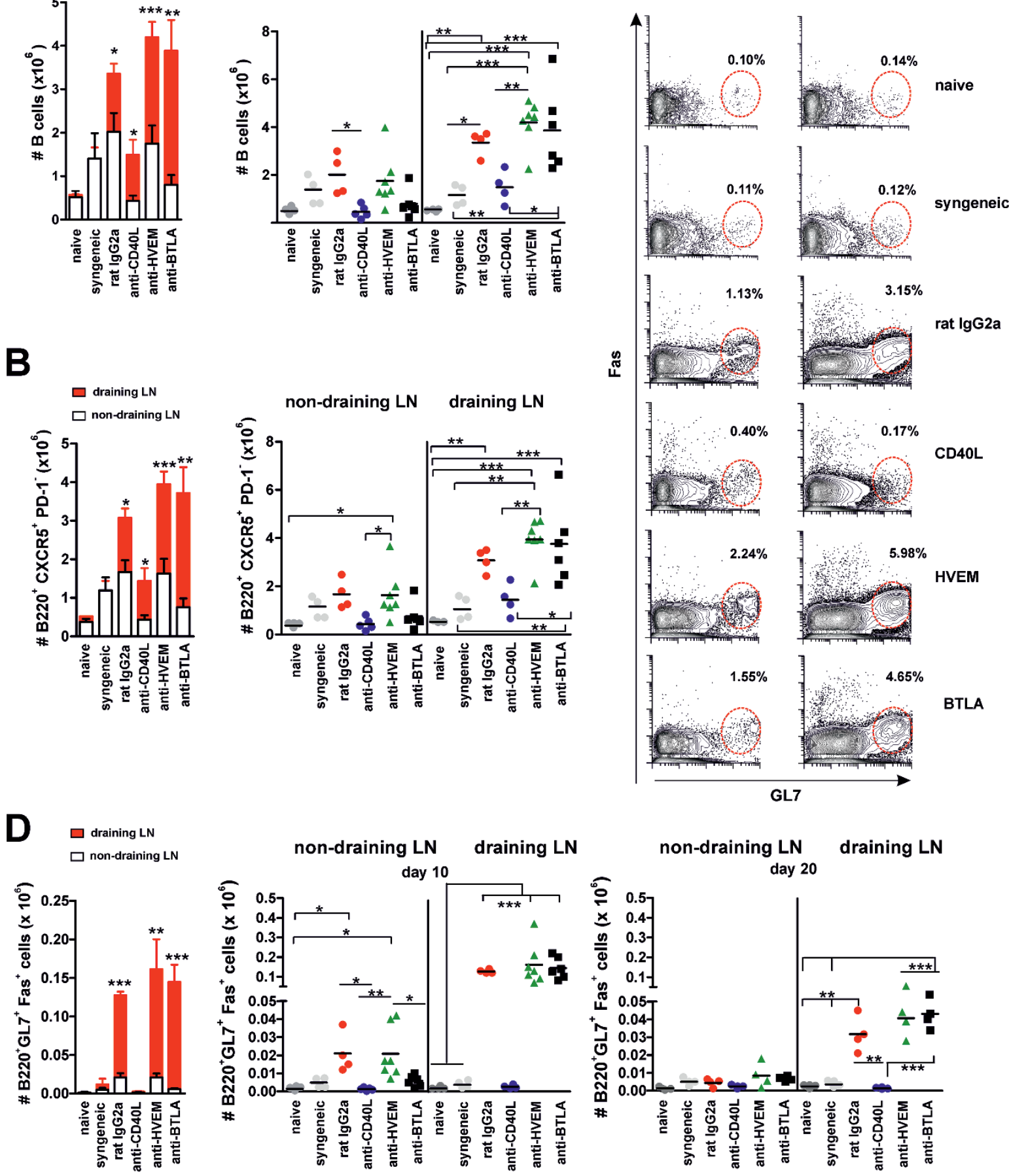

Figure 2 GC cell expansion proceeds normally despite HVEM/BTLA interaction blockade. The absolute B-cell numbers in the non-draining and draining LNs were calculated at day 10 post transplantation (a, left panel). The absolute B-cell numbers in the non-draining and draining LNs were compared among the different experimental groups (a, right panel). (b) The same general description as in Figure 2a applies for the follicular B cells $\left(\mathrm{B}_{22} \mathrm{C}^{+} \mathrm{CXCR}^{+} \mathrm{PD}^{-} \mathbf{1}^{-}\right.$). (c) The GC B-cell expansion was identified as a B-cell population expressing B220, Fas, and GL7 ${ }^{+}$in the draining and nondraining LNs of the experimental groups. (d) The same general description as in Figure 2a applies for GC B cells expressing GL7 and Fas at day 10 (left and middle panel) and at day 20 (right panel) post transplantation of BALB/c skin onto C57BL/6 recipients. The results are displayed as the means \pm SEM. Statistical significance and $P$-values were calculated using unpaired Student's $t$ test for the comparison of means between the draining versus non-draining LNs of each experimental group, whereas the one-way analysis of variance was applied for the comparison of the means among the experimental groups with regard to the draining or non-draining LNs. The statistical significance criteria were as follows: $* P<0.05$; $* * P<0.005$; ***P<0.0005. These plots display data that were pooled from three independent experiments. BTLA, B-and T-lymphocyte attenuator; $\mathrm{GC}$, germinal centre; HVEM, herpesvirus entry mediator. 
Treatment with anti-BTLA mAb upregulates HVEM expression on follicular and extrafollicular allogeneic $\mathrm{Th}$ cells after skin graft transplantation

We evaluated the membrane-bound BTLA and HVEM expression levels on follicular and extrafollicular $\mathrm{T}$ helper cells during the acute phase of rejection after allogeneic skin transplantation in non-draining and draining LNs. Treatment with anti-CD40L or anti-HVEM antibodies in comparison with naïve and syngeneic animals as well as those treated with the isotype control did not lead to changes in the BTLA expression levels on the Tfh cells $\left(\mathrm{CXCR}^{+}{ }^{\mathrm{PD}-1^{+}}\right.$, Figure $3 \mathrm{a}$, Top panel) or the non-Tfh cells (extrafollicularCXCR $5^{-} \mathrm{PD}-1^{+}$, medium panel and extrafollicular $\mathrm{CXCR}^{-} \mathrm{PD}-1^{-}$, bottom panel), as determined by the mean fluorescence intensity value measurements (Figure 3a). The BTLA expression could not be followed in anti-BTLA-treated mice because, as previously reported, anti-BTLA mAb treatment down-modulates the receptor, which hinders its detection by flow cytometry (Figure 3a). ${ }^{21}$

HVEM expression was also monitored by flow cytometry thanks to the anti-HVEM mAb, clone 5A8, which does not compete with the in vivo-administered anti-HVEM mAb, clone $6 \mathrm{C} 9$, for binding to cell-bound HVEM. ${ }^{20}$ As illustrated in Figure 3b, the HVEM expression level was significantly enhanced in the anti-BTLA-treated mice in both the Tfh cells (top panel) and the extrafollicular T helper cells (CXCR5 ${ }^{-} \mathrm{PD}-1^{+}$, medium panel; $\mathrm{CXCR}^{-} \mathrm{PD}^{-} 1^{-}$, bottom panel) compared with the HVEM levels in most of the experimental groups. This denotes that disrupting the intrinsic cis-interaction between HVEM and BTLA by antibody-mediated down-modulation of BTLA unblocks HVEM, leading to significantly augmented expression. This phenomenon occurred in non-draining and draining LNs, suggesting that this change of in HVEM expression was due to the anti-BTLA mAb treatment rather than to activation in response to the alloantigen stimulation.

ICOS expression was also tracked during the acute phase of rejection. Much higher expression levels of this molecule on the Tfh cell surfaces were observed. Additionally, intermediate ICOS expression levels were observed on the $\mathrm{PD}-1^{+}$extrafollicular T helper cells and a lack of detectable ICOS staining was observed in the extrafollicular population of $\mathrm{CD}^{+} \mathrm{PD}^{-} \mathrm{T}$ cells (Figure $3 \mathrm{c}$ ).

Overall, these observations indicate that the intrinsic cisinteraction between HVEM and BTLA was disturbed with the administration of the anti-BTLA mAb in the alloreactive Tfh cells and the non-Tfh cells, which led to enhanced HVEM expression.

\section{The allogeneic host antidonor humoral immune response was unaffected despite the antibody-mediated blockade of the BTLA/HVEM-interacting pathway}

Although the clonal expansion of the CD4Tfh cells and GC B cells in response to the allogeneic stimulation was unaltered despite the HVEM/BTLA interaction blockade, we decided to evaluate whether the antibody-mediated response was slightly augmented similar to BTLA-deficient mice that were immunized with ovalbumin plus adjuvant. ${ }^{35}$ Two experimental approaches were adopted depending on the alloantigen source and the immunological technique that was chosen (membranebound $\mathrm{H}-2^{\mathrm{d}}$ class I alloantigens expressed on the cell surface of $\mathrm{BALB} / \mathrm{c}$ thymocytes for flow cytometry or recombinant-soluble biotinylated $\mathrm{H}-2 \mathrm{~K}^{\mathrm{d}}$ and $\mathrm{H}-2 \mathrm{~K}^{\mathrm{b}}$ monomers for ELISA), and they were used to follow the allogeneic humoral immune responses. The first strategy consisted of incubating diluted serum samples collected from recipient mice at days 10 and 20 post transplantation with donor $(\mathrm{BALB} / \mathrm{c})$ thymocytes. Then, the host antidonor IgM and $\operatorname{IgG}$ isotype-specific humoral responses were analysed (Figure 4a-e). No significant differences in the donor-specific antibody response in the transplanted mice treated with isotype control or the anti-HVEM or anti-BTLA $\mathrm{mAbs}$ were seen for any of the antidonor isotype-specific antibodies ( $I g M, I g G_{3}, I g G_{1}, \operatorname{IgG}_{2 \mathrm{a}}$ and $\operatorname{IgG}_{2 \mathrm{~b}}$ ), which were assessed by flow cytometry, except for the anti-CD40L mAb-treated group mice, in which the humoral responses were completely abolished. We also took advantage of biotinylated $\mathrm{K}^{\mathrm{b}}$ recombinant monomers (syngeneic) and $\mathrm{K}^{\mathrm{d}}$ recombinant monomers (allogeneic) to monitor the humoral immune response at days 0,10 and 20 after transplantation to detect the presence of thymus-dependent IgG-mediated immune responses (IgG, allogeneic $\mathrm{H}-2 \mathrm{~K}^{\mathrm{d}}$, top panel and $\mathrm{IgG}$, syngeneic $\mathrm{H}-2 \mathrm{~K}^{\mathrm{b}}$, bottom panel) (Figure 4f). The same outcome as that observed in the flow cytometry was observed in the ELISAs for the donorspecific total IgG antibodies. As expected, the transplanted mice that were treated with anti-CD40L mAb were unable to mount a proper host antidonor humoral IgG-mediated immune response (Figure $4 \mathrm{a}-\mathrm{f}$ ). BALB/c skin allografts in the B6 recipients were rejected following a similar kinetics as the isotypetreated controls despite the antibody targeting the HVEM/ BTLA interactions. All BALB/c allogeneic skin grafts in B6 recipients were rejected by day 11 in the recipient mice that were either treated with the rat $\operatorname{IgG}_{2 \mathrm{a}}$ isotype control or the anti-BTLA or anti-HVEM antibodies. However, the antiCD40L-treated group showed a modest but significant prolongation in graft survival (days 13-14) (Figure 4g). In summary, the thymus-dependent and thymus-independent humoral immunities were unaltered despite disabling the molecular interaction between HVEM and BTLA.

As the expression of BTLA differs in distinct mouse strains, ${ }^{21,36}$ we decided to evaluate the antidonor humoral immune response and the fate of fully MHC-mismatched allogeneic skin grafts in an alternative mouse strain combination (donor $\mathrm{B} 6$ to $\mathrm{BALB} / \mathrm{c}$ recipient mice) that was treated with antibodies that interfered with the BTLA/HVEM pathway. The host antidonor humoral immune response in the BALB/ c recipients was delayed compared with the B6 recipients at day 10 after skin grafting, but they reached similar levels at day 20. Neither the anti-BTLA mAb nor the anti-HVEM $\mathrm{mAb}$ treatment affected the course of the allogeneic humoral immune responses (Figure 5a). The fate of the fully MHCmismatched B6 allogeneic skin grafts that were placed in the Balb/c mice was only significantly delayed after anti-CD40L treatment; however, no effect was seen as a consequence of targeting the HVEM/BTLA pathway (Figure 5b). 

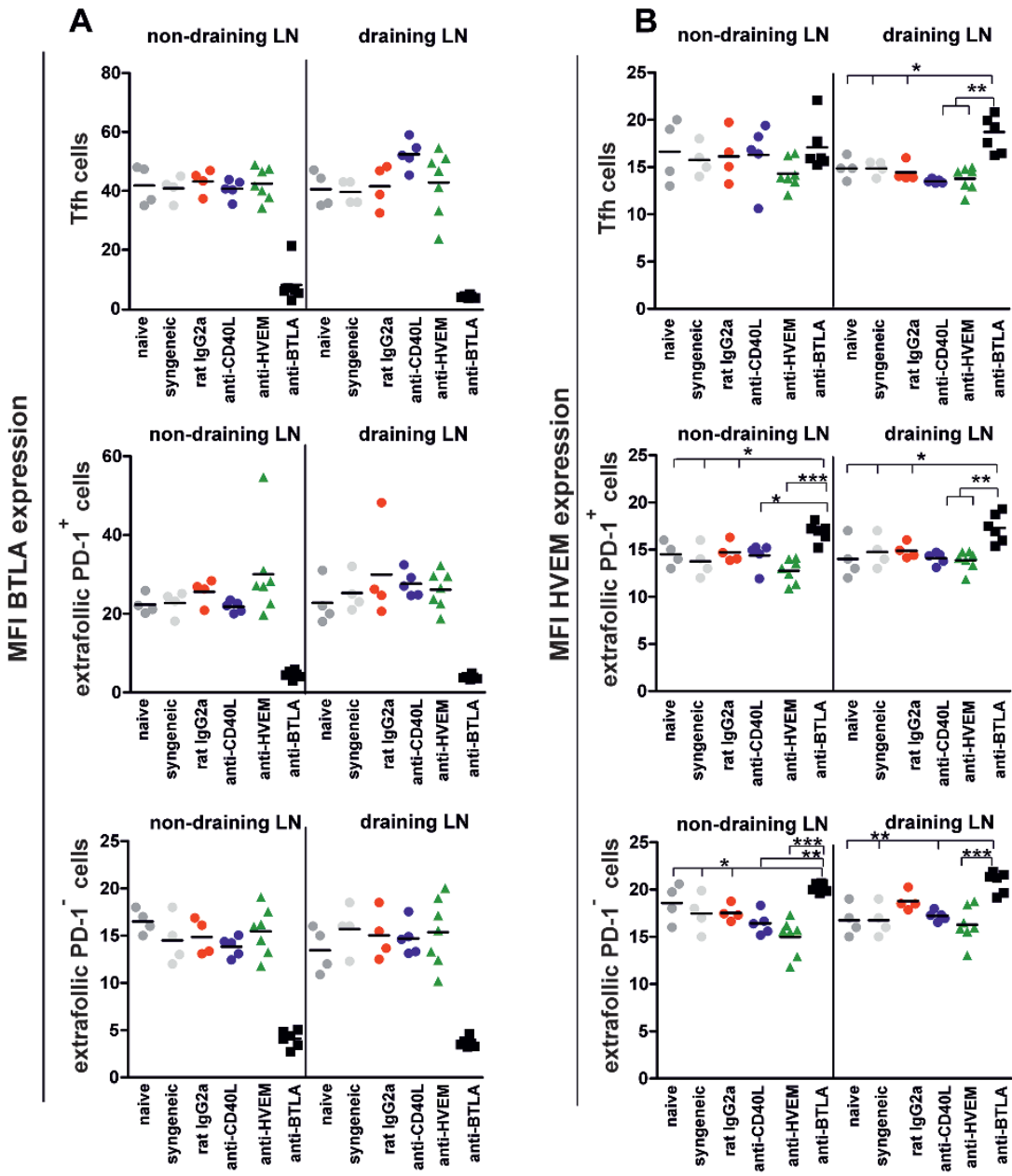

C

non-draining LN
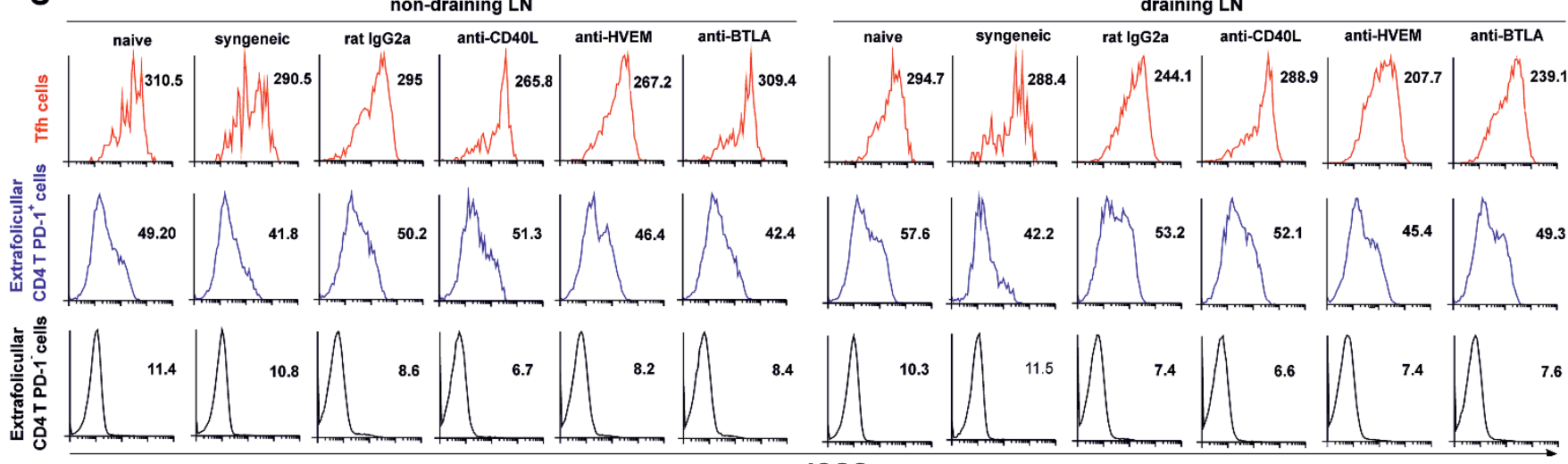

Figure 3 The anti-BTLA antibody treatment disables the BTLA/HVEM cis-complex and, consequently, HVEM expression increases on Tfh cells and on extrafollicular helper cells during the allogeneic humoral immune responses. Axillar and brachial non-draining and draining LNs from B6 recipient mice that were transplanted with $\mathrm{BALB} / \mathrm{c}$ allogeneic skin were collected on day 10 . The BTLA expression levels on the $\mathrm{CXCR} 5^{+} \mathrm{Tfh}$ cells (a, upper panel), the $\mathrm{CXCR} 5^{-}$extrafollicular $\mathrm{CD} 4^{+} \mathrm{PD}^{+}$cells (a, middle panel) and the extrafollicular $\mathrm{CD}^{+} \mathrm{PD}^{-}$(a, lower panel) $\mathrm{T}^{-}$cells are shown. CXCR5 ${ }^{+}$Tfh cells (b, upper panel), CXCR5 ${ }^{-}$extrafollicular CD4 ${ }^{+}$PD- $1^{+}$(b, middle panel) and CXCR5 ${ }^{-}$extrafollicular CD4 ${ }^{+}$PD- $1^{-}$ (b, lower panel) T cells expressing HVEM are also represented. (c) ICOS expression on T cells costimulates B cells that express ICOS-L during the humoral immune responses and contributes to the germinal centre formation. The ICOS expression level was analysed on Tfh cells and extrafollicular helper cells in the non-draining and draining LNs of each experimental group. The mean fluorescence intensity values of the ICOS expression on the Tfh and extrafollicular T cells are depicted. The one-way analysis of variance was applied for the comparison of the means among groups and the following criteria for significance were used: $* P<0.05 ; * * P<0.005$; *** $P<0.0005$. BTLA, B- and T-lymphocyte attenuator; HVEM, herpesvirus entry mediator; Tfh, T follicular helper cells. 


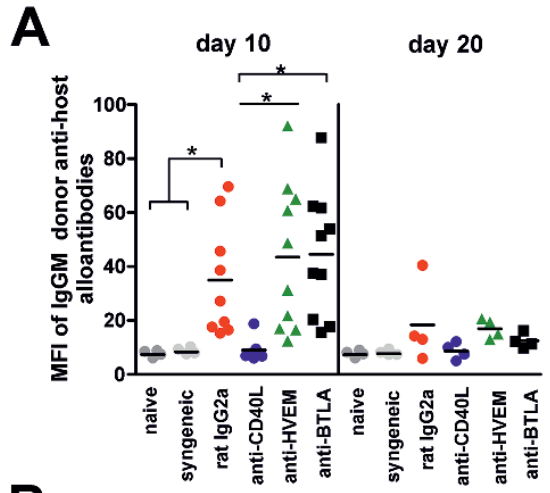

B
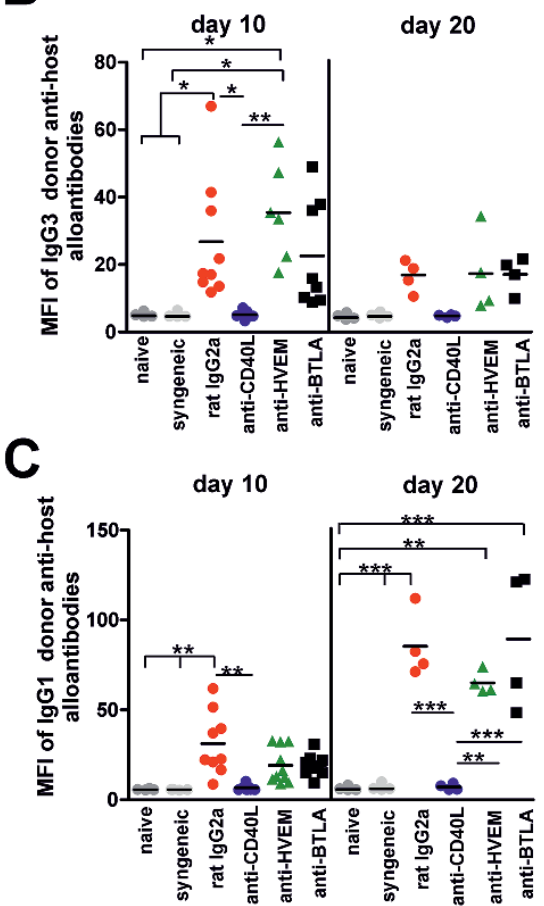

D

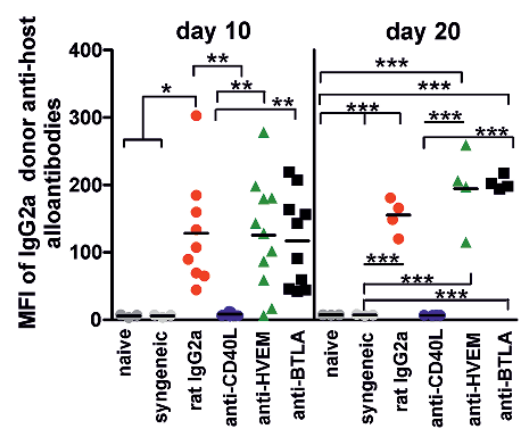

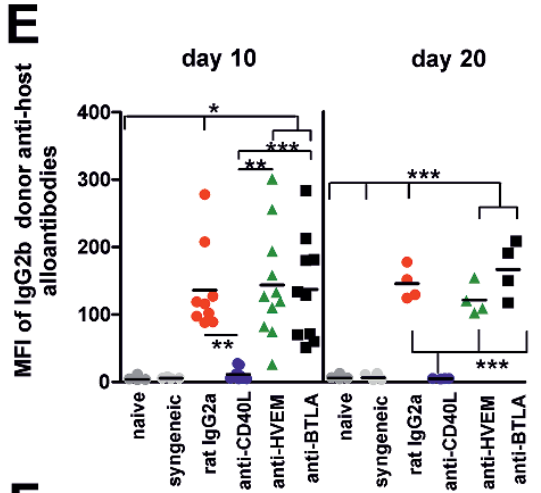

$\mathbf{F}$

anti-K ${ }^{\mathrm{d}}$ ELISA (total IgG)

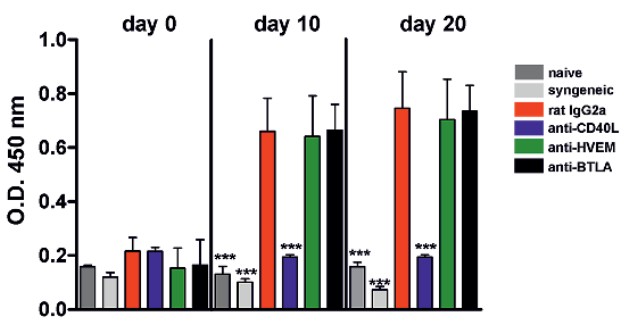

anti-K $K^{b}$ EISA (total IgG)

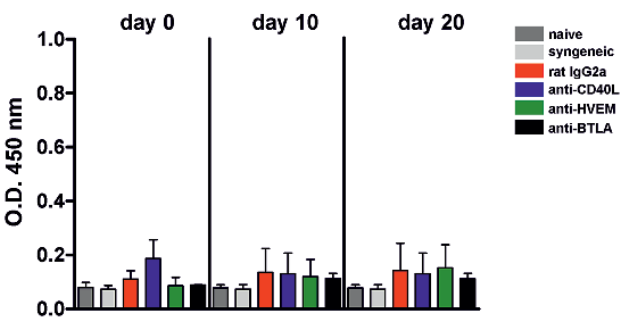

G

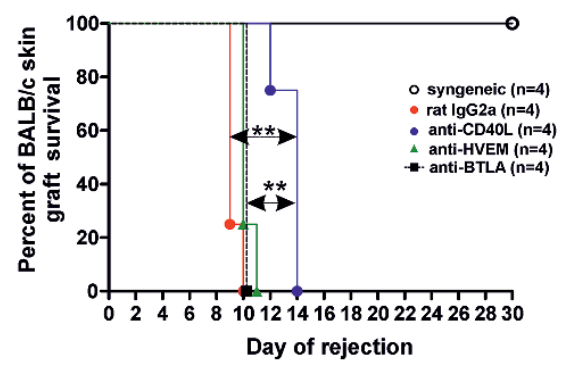

Figure 4 The donor-specific allogeneic immune response was not dependent on the HVEM/BTLA signalling pathway. Sera from B6 recipient mice that were transplanted with BALB/c skin were collected on days 10 and 20 after transplantation. The samples were then incubated with donor thymocytes and the host antidonor thymic-independent $\operatorname{lgM}$ and $\lg _{3}\left(\mathbf{a}\right.$ and $\mathbf{b}$ ) and the thymic-dependent $\lg G_{1}$, $\operatorname{lgG}_{2 a}$ and $\operatorname{lgG}_{2 b}$ humoral immune response levels (c-e) were analysed by flow cytometry and represented as the mean fluorescence intensity. (f) To specifically detect the host IgG antidonor-specific antibodies that were present in the serum of mice that were transplanted with BALB/c skin, an ELISA using $K^{d}$ (allogeneic) and $K^{b}$ (syngeneic) monomers was also applied. (g) The survival of BALB/c skin allografts that were placed on the B6 recipients was monitored overtime in distinct experimental groups. The data are the means \pm SD of five to seven mice. The statistical significance and $P$ values were calculated as indicated in the Materials and Methods section. BTLA, B- and T-lymphocyte attenuator; HVEM, herpesvirus entry mediator. 


\section{DISCUSSION}

Antibody-mediated rejection is one of the major challenges in the field of solid-organ transplantation due to its contribution in the insidious pathology of chronic graft rejections that are refractory to current maintenance immunosuppressive drugs. $^{2-4}$ There is a need to gain insight into the molecular mechanisms that are responsible for the exchange of information between Tfh cells and B cells to modulate the humoral immune response, particularly in autoimmune diseases and antibody-mediated graft rejections. The molecular pathways that are involved in the exchange of information between Tfh cells and B cells through cell surface molecules of the TNF/TNFR family are essential immune checkpoints that dampen T-/B-cell collaborations and prevent antibody-mediated allogeneic immune responses. Recombinant fusion proteins and mAbs (biologics) that target members of the TNF, TNFR and immunoglobulin superfamily molecules are the central focus of attention for therapy in many immune-mediated diseases. ${ }^{37,38}$ An example of this statement is the efficacy of CD40L/CD40 targeting in preclinical and clinical settings of transplantation and autoimmune disease. ${ }^{39,40}$

The data presented herein demonstrate that modulation of BTLA/HVEM pathway does not affect Tfh cell expansion, GC reactions or the de novo formation of host antidonor allogeneic antibodies. BTLA, like other members of the immunoglobulin superfamily, such as CTLA4 and PD1, functions as a co-inhibitory receptor of the immunoglobulin superfamily. The engagement of BTLA and CD160 by HVEM induces a co-inhibitory signal through the recruitment of the SHP-1 and SHP-2 phosphatases, which are able to attenuate the functional activity of tyrosine kinases that are mobilized upon T-cell receptor recognition of antigens. ${ }^{23,41-44}$ The HVEM/BTLA cis-interaction (where both molecules are expressed on the same cell) is an intrinsic co-inhibitory mechanism that prevents HVEM or BTLA from interacting in the trans configuration (the two interacting molecules are in different cells). This cis-interaction keeps naïve T cells in a resting state. Upon T-cell activation, transient expression of LIGHT disrupts BTLA/ HVEM cis-interactions, which allows for simultaneous HVEM/BTLA coinhibitory and HVEM/LIGHT costimulatory trans interactions to occur as the binding site of BTLA on HVEM (CRD1) is different from the binding site of LIGHT (CRD2-3), which are on the opposite sites of the molecule. ${ }^{45}$

Similar to LIGHT, the administration of anti-BTLA mAb seems to contribute to HVEM/BTLA molecular instability and subsequent disassembling of the HVEM/BTLA complex, leading to enhanced HVEM expression in both draining (ongoing alloreactive response) and non-draining (resting non-inflammatory response) LNs. This enhanced HVEM expression was independent of the activation status (draining versus non-draining) and could be attributed to only the antiBTLA mAb treatment that permitted better exposure of HVEM for detection by anti-HVEM antibodies, as illustrated in Figure $3 \mathrm{~b}$. The anti-BTLA mAb treatment consistently and significantly augmented the HVEM expression level not only on
A anti-K $K^{b}$ EISA (total IgG)

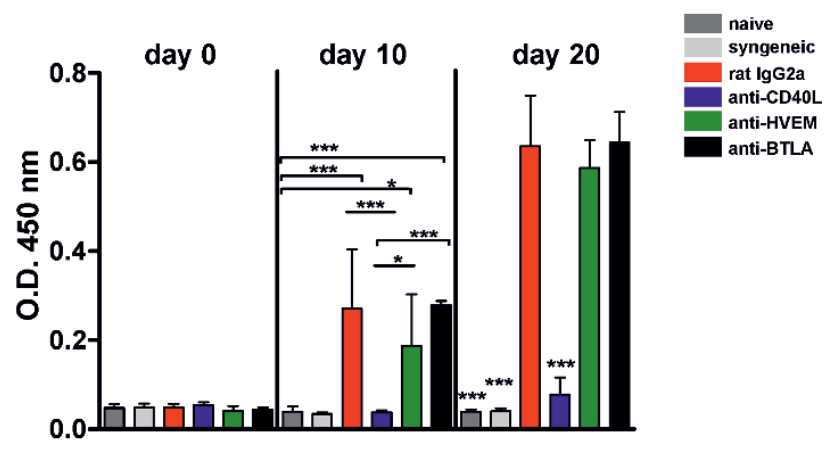

anti-K $K^{\mathrm{d}}$ ELISA (total IgG)
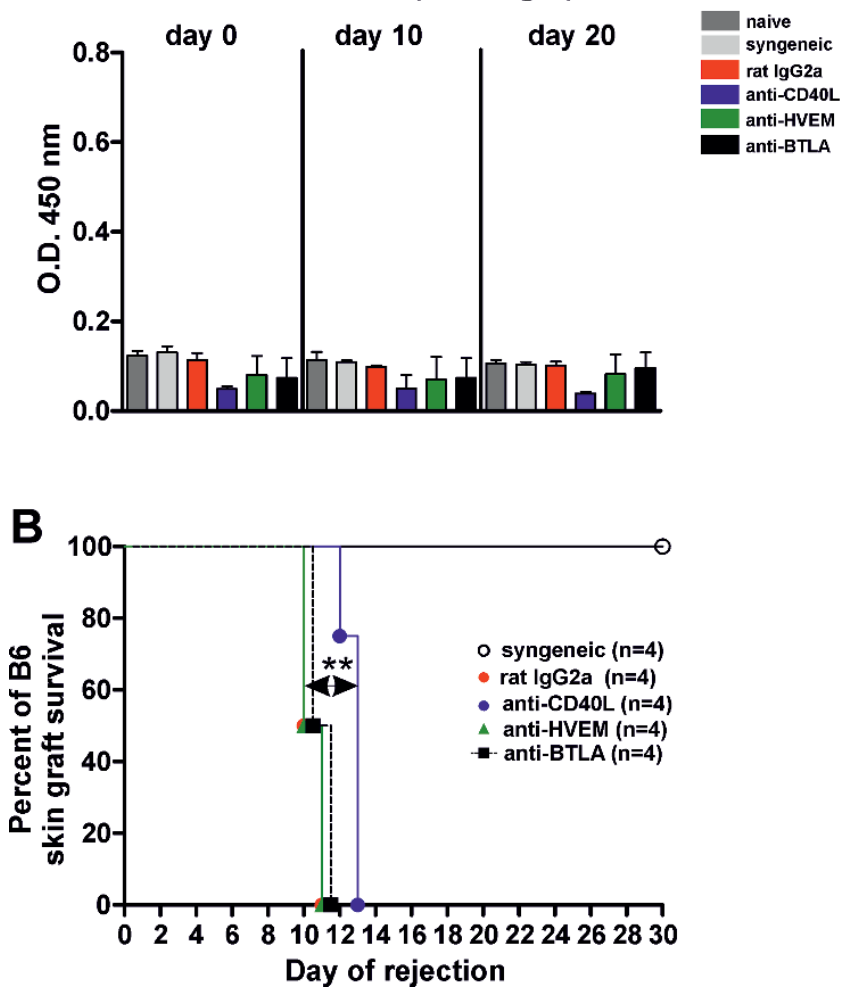

Figure 5 Targeting BTLA/HVEM did not modulate the graft rejection course in $B A L B / c$ recipients that were grafted with allogeneic $B 6$ skin. (a) A sandwich ELISA was used to monitor the host IgG antidonorspecific antibodies in fully major histocompatibility complexmismatched transplanted BALB/C recipients of $\mathrm{B} 6$ skin allografts. The data are the means \pm SD of four mice. (b) The B6 skin graft survival was monitored over time in syngeneic controls and allogeneic experimental groups, and the survival curves were calculated using the Kaplan-Meier life table method. Statistical analysis was performed with the long rank test. Statistical significance is indicated as follows: ${ }^{*} P<0.05$; $* * P<0.005 ; * * * P<0.0005$.BTLA, B- and T-lymphocyte attenuator; HVEM, herpesvirus entry mediator.

Tfh cells but also on extrafollicular $\mathrm{T}$ helper cells in both the draining and non-draining LNs. Our data are in agreement with the hypothesis put forward by Ware et al, which states that some antibodies, such as the anti-BTLA clone used in this work, recognize epitopes in the HVEM/BTLA binding site and cause molecular complex instability of the BTLA/HVEM cis-complex 
making HVEM more accessible to detection by anti-HVEM antibodies. $^{22}$

Remarkably, the anti-BTLA treatment reduced the B-cell numbers in non-draining LNs. To account for this observation, the most straightforward interpretation is that engagement of BTLA by HVEM in the cis-configuration is required for resting naïve B-cell homeostasis. BTLA signalling might contribute to the survival and control of B-cell numbers under noninflammatory conditions. Additional experimental evidence supporting the role of BTLA in the survival of lymphoid cells was shown with the adoptive transfer of semiallogeneic BTLAdeficient splenocytes into $\mathrm{F} 1$ recipients, which leads to an early expansion of alloreactive effector $\mathrm{T}$ cells followed by a rapid decay. This observation of impaired donor alloreactive BTLAdeficient T-cell survival during the course of Graft versus Host Disease (GvHD), which promotes the pathology resolution, indicates that BTLA may also play a role in alloreactive T-cell survival. ${ }^{46}$ The BTLA/HVEM interaction is also essential for the homeostatic control of $\mathrm{CD} 8 \alpha^{-}$dendritic cell numbers. Indeed, HVEM- and BTLA-deficient mice displayed an increased number of $\mathrm{CD} 8 \alpha^{-}$dendritic cells. De Trez et al showed that the engagement of LT $\beta$ R by LT $\alpha \beta$, and to a lesser extent by LIGHT (the other TNF ligand of LT $\beta$ R), functions as a growth regulator that controls $\mathrm{CD} 8 \alpha^{-}$DC numbers in the spleen, ${ }^{47}$ although this growth-promoting effect could be counterbalanced by the inhibitory HVEM/BTLA signalling pathway. Altogether, these observations provide experimental evidence for the implication of HVEM/BTLA interactions in the homeostatic control of cell numbers in different subpopulations of immune cells.

Most of the evidence regarding the role of BTLA in humoral responses comes from the analysis of BTLA-deficient mice that are prone to the development of humoral-mediated autoimmune diseases as they age. ${ }^{23-25}$ The humoral immune response and propensity to develop autoimmune diseases are enhanced not only in BTLA-deficient mice $e^{23,35}$ but also in HVEM-deficient mice. ${ }^{48}$ The latter strengthens the predominant in vivo function of HVEM as an inhibitory ligand rather than a costimulatory ligand. The HVEM-deficient mice, like the BTLA-deficient mice, are more predisposed to develop experimentally induced autoimmune diseases than their wild-type (WT) counterparts, likely due to their inability to co-inhibit activated T cells through BTLA ${ }^{48}$ and the impaired suppressive activity of $\mathrm{T}$ regulatory cells (Tregs) that lack HVEM in the control of effector T cells expressing BTLA. ${ }^{49}$ These observations are in favour of the notion that BTLA may function as a co-inhibitory receptor on CD4 Tfh cells, which contributes, to a certain extent, to the regulation of humoral-mediated immune responses. ${ }^{23}$ The other evidence in the literature that supports the role of the BTLA/HVEMinteracting pathway in humoral immune responses is based on a mouse model in which BTLA KO TcR transgenic anti-OVA (ovalbumin) $\mathrm{CD}^{+}{ }^{+} \mathrm{T}$ cells were ex vivo-stimulated under Tfh cell-inducing conditions (Tfh-like cells) and then adoptively transferred into WT mice that were immunized with OVA and adjuvant. In this setting, more antigen-specific $\operatorname{IgG}_{2 \mathrm{a}}$ and $\operatorname{IgG}_{2 \mathrm{~b}}$ production was observed in the recipient mice that were adoptively transferred with Tfh-like BTLA-deficient cells than in the recipient mice that were adoptively transferred with WT Tfhlike cells. ${ }^{35}$ Although the development of Tfh cells was not affected in the BTLA-deficient mice, their function was slightly augmented and, consequently, the production of antigenspecific antibodies was also slightly increased. ${ }^{35}$

It will be necessary to reconcile the discrepancies of the slightly enhanced antibody response in autoimmune diseases and after conventional immunization of BTLA-deficient mice with the results of our investigation in the setting of fully MHCmismatched allotransplantation. One explanation that may account for the lack of the role for BTLA/HVEM interactions in our experimental setting is that antibody treatment does not affect Tfh T-cell numbers, which is in agreement with the observed normal development of Tfh cells in BTLA-deficient mice. ${ }^{35}$ Another possibility is that inborn deficiency of BTLA or HVEM, which remains during development into adulthood, may affect the survival and accumulation of Tfh or $\mathrm{T}$ follicular regulatory cells (Tfreg) in the secondary lymphoid organs and their function. ${ }^{50}$ For instance, a decrease in the frequency of Treg numbers due to compromised survival in BTLA-deficient mice would undermine the counter-regulation of T-helper-mediated B-cell responses, which would lead to an increased antibody response in BTLA-deficient mice compared with WT mice. In agreement with the concept that BTLA functions as a prosurvival molecule for T cells is the fact that the engagement of BTLA on donor alloreactive $\mathrm{T}$ cells is required for their survival in a graft versus host disease murine model. ${ }^{46,51}$ Thus, a single injection of an agonistic anti-BTLA antibody (clone 6A6) at the time of allogeneic bone marrow transplantation was sufficient to prevent GvHD through a mechanism that was mediated by donor Tregs. ${ }^{51,52}$ The agonistic effect of the anti-BTLA antibody treatment and not the blockade of the interaction of HVEM/ BTLA on donor CD4 effector cells promoted a rapid contraction of the CD4 T-cell-mediated allogeneic immune response due to impaired survival of donor $\mathrm{T}$ cells expanding in response to lymphopenia in conditioned hosts and to an advantageous and competitive homeostatic expansion of Tregs that were responsible for prevention of the disease. ${ }^{51}$ Tregs have also been implicated in the regulation of the allogeneic response in CBA mice receiving a heart allograft from $\mathrm{B} 6$ donors treated with an agonistic anti-BTLA antibody. This regulation was mediated by an IL-10-dependent mechanism of suppression. ${ }^{53}$

The intervention with two antibodies against the HVEM/ BTLA pathway, including the anti-HVEM mAb (clone 6C9), which is capable of blocking BTLA/HVEM interactions but not HVEM/LIGHT interactions ${ }^{20}$ and the anti-BTLA mAb (clone $4 \mathrm{G} 12 \mathrm{~b}$, which is a non-depleting, agonist antibody capable of attenuating in vivo graft versus host disease), did not affect the allogeneic humoral immune response. ${ }^{27,54}$ This result means that neither BTLA co-inhibition nor the HVEM/BTLA blockade influenced the course of the alloreactive antibody response. Although the outcome in the transplantation setting is different 
from that observed in autoimmune disease in BTLA-deficient mice, we have to acknowledge that the predisposition to antibody-mediated autoimmune disease is an indolent slow moving pathology in BTLA-deficient mice that occurs late in life. ${ }^{23}$ Moreover, enhanced humoral responses were demonstrated in BTLA-deficient mice that were immunized with OVA plus adjuvant, which was done in a transgenic mouse model that included a high frequency of antigen-specific monoclonal $\mathrm{T}$ cells, and after ex-vivo generation of Tfh cells that were then adoptively transferred into WT recipients, which forces the experimental system to conditions that are beyond normal physiology. ${ }^{35}$

The kinetics of allogeneic skin graft rejection across a fully MHC-mismatched barrier was similar in the $\mathrm{B} 6 \mathrm{and} \mathrm{Balb} / \mathrm{c}$ recipients regardless of the administration of the anti-BTLA or antiHVEM antibodies compared with the isotype control. The most remarkable finding was a delay in the production of host antidonor IgG antibodies in the $\mathrm{Balb} / \mathrm{c}$ recipients of the $\mathrm{B} 6$ skin grafts compared with the $\mathrm{B} 6$ recipients of the Balb/c skin grafts, although this did not alter the skin graft rejection course.

We have confirmed the robust inhibitory activity of CD40L/CD40 blockade on GC formation. This seems to be due to the dual effector functional activity of the anti-CD40L $\mathrm{mAb}$ (clone MR1, hamster IgG). The therapeutic administration of this antibody antagonizes CD40L/CD40 interactions, but more importantly, it also depletes recently activated alloreactive T cells expressing CD40L and alloreactive Tfh cells, which consequently abrogates T-/B-cell collaborations and antibody production. ${ }^{29,30}$ The antibodies used in this study that were raised against BTLA/HVEM pathway molecules were both rat $\operatorname{IgG}_{2 \mathrm{a}}$ isotype antibodies. Rat $\operatorname{IgG}_{2 \mathrm{a}}$ isotype antibodies do not efficiently bind to the mouse Fc $\gamma$ RI (CD64) and Fc $\gamma$ RIV (CD16.2) proteins, which are the two major Fc receptors involved in antibody-dependent cellular cytotoxicity that is mediated by natural killer cells and myeloid cells $s^{55}$ ( Li F and Ravetch JV, 2015). Thus, this result rules out the possibility that antibody treatment might have driven any functional activity through depletion. Furthermore, the BTLA/HVEM interactions were properly modulated because the receptors were saturated and an excess of antibody against these receptors was present in the serum of the treated mice over time after a single administration of $1 \mathrm{mg} / \mathrm{mouse}(40 \mathrm{mg} / \mathrm{kg})$ at the time of transplantation. Finally, despite the large numbers of hybridoma clones that secrete antibodies against mouse BTLA that are available in the market, they all detect BTLA expression on B cells well; however, their BTLA staining on $\mathrm{T}$ cells is negative or weak. ${ }^{36}$ This accounts for the impossibility to follow BTLA expression after treatment with an anti-BTLA mAb.

To understand the mechanism that accounts for the subtly enhanced humoral immunity observed in the BTLA- and HVEM-deficient mice, the Tfh cell, follicular Treg and B-cell survival and functions need to be further investigated in inflammatory mouse models of disease in which the humoral response contributes to the disease pathology. Thus, it will be possible to gain insight into the molecular mechanisms that guide humoral immunity and determine the potential modulatory activity of the HVEM/BTLA-interacting pathway for therapeutic purposes.

In conclusion, the therapeutic blockade of the HVEM/BTLA pathway did not alter the clonal expansion of the Tfh cells or GC B cells that were responsible for the GC reaction or the subsequent production of donor-specific alloantibodies following transplantation.

\section{COMPETING INTEREST}

The authors declare no conflicting financial interests.

\section{ACKNOWLEDGEMENTS}

This work was supported by grants from the Spanish Ministry of Health (Fondo de Investigaciones Sanitarias, PI13/00029), Department of Education of Castilla and Leon Regional Government (Grant\# LE093U13) and the Mutua Madrileña Foundation (Basic research grants 2012) to J.I.R.B. Additional support was provided by Miguel Servet National Program (Ministry of National Health) CP12/03063 and by Gerencia Regional de Salud GRS963/A/2014 to M.L.R.G. We are particularly grateful to Mr. Leonides Alaiz for the outstanding animal husbandry.

Supplementary Information accompanies the paper on the Cellular \& Molecular Immunology's website (http://www.nature.com/cmi/).

1 Colvin RB, Smith RN. Antibody-mediated organ-allograft rejection. Nat Rev Immunol 2005; 5: 807-817.

2 Smith RN, Colvin RB. Chronic alloantibody mediated rejection. Semin Immunol 2012; 24: 115-121.

3 Willicombe M, Roufosse C, Brookes P, McLean AG, Galliford J, Cairns $T$ et al. Acute cellular rejection: impact of donor-specific antibodies and C4d. Transplantation 2014; 97: 433-439.

4 Bamoulid J, Staeck O, Halleck F, Khadzhynov D, Brakemeier S, Durr $\mathrm{M}$ et al. The need for minimization strategies: current problems of immunosuppression. Transp/ Int 2015; 28(8): 891-900.

5 Steele DJ, Laufer TM, Smiley ST, Ando Y, Grusby MJ, Glimcher LH et al. Two levels of help for B cell alloantibody production. J Exp Med 1996; 183: 699-703.

6 Conlon TM, Saeb-Parsy K, Cole JL, Motallebzadeh R, Qureshi MS, Rehakova $\mathrm{S}$ et al. Germinal center alloantibody responses are mediated exclusively by indirect-pathway CD4 T follicular helper cells. J Immunol 2012; 188: 2643-2652.

7 de Graav GN, Dieterich M, Hesselink DA, Boer K, Clahsen-van Groningen MC, Kraaijeveld $\mathrm{R}$ et al. Follicular $\mathrm{T}$ helper cells and humoral reactivity in kidney transplant patients. Clin Exp Immunol 2015; 180: 329-340.

8 Claman HN, Chaperon EA, Triplett RF. Thymus-marrow cell combinations. Synergism in antibody production. Proc Soc Exp Biol Med 1966; 122: 1167-1171.

9 Mitchell GF, Miller JF. Cell to cell interaction in the immune response. II. The source of hemolysin-forming cells in irradiated mice given bone marrow and thymus or thoracic duct lymphocytes. J Exp Med 1968; 128: 821-837.

10 Ramiscal RR, Vinuesa CG. T-cell subsets in the germinal center. Immunol Rev 2013; 252: 146-155.

11 Yu D, Batten M, Mackay CR, King C. Lineage specification and heterogeneity of T follicular helper cells. Curr Opin Immunol 2009; 21: 619-625.

12 Vogelzang A, McGuire HM, Yu D, Sprent J, Mackay CR, King C. A fundamental role for interleukin-21 in the generation of $\mathrm{T}$ follicular helper cells. Immunity 2008; 29: 127-137. 
13 Crotty S. T follicular helper cell differentiation, function, and roles in disease. Immunity 2014; 41: 529-542.

14 Nurieva RI, Chung Y, Martinez GJ, Yang XO, Tanaka S, Matskevitch TD et al. Bcl6 mediates the development of $\mathrm{T}$ follicular helper cells. Science 2009; 325: 1001-1005.

15 Kelsoe G. The germinal center reaction. Immunol Today 1995; 16: 324-326.

16 De Silva NS, Klein U. Dynamics of B cells in germinal centres. Nat Rev Immunol 2015; 15: 137-148.

17 Forster R, Mattis AE, Kremmer E, Wolf E, Brem G, Lipp M. A putative chemokine receptor, BLR 1 , directs $B$ cell migration to defined lymphoid organs and specific anatomic compartments of the spleen. Cell 1996; 87: 1037-1047.

18 Cheung TC, Humphreys IR, Potter KG, Norris PS, Shumway HM, Tran $\mathrm{BR}$ et al. Evolutionarily divergent herpesviruses modulate $\mathrm{T}$ cell activation by targeting the herpesvirus entry mediator cosignaling pathway. Proc Natl Acad Sci USA 2005; 102: 13218-13223.

19 Mauri DN, Ebner R, Montgomery RI, Kochel KD, Cheung TC, Yu GL et al. LIGHT, a new member of the TNF superfamily, and lymphotoxin alpha are ligands for herpesvirus entry mediator. Immunity 1998; 8 21-30.

20 Del Rio ML, Jones ND, Buhler L, Norris P, Shintani Y, Ware CF et al. Selective blockade of herpesvirus entry mediator-B and T lymphocyte attenuator pathway ameliorates acute graft-versus-host reaction. J Immunol 2012; 188: 4885-4896.

21 Del Rio ML, Kaye J, Rodriguez-Barbosa JI. Detection of protein on $B T L A($ low) cells and in vivo antibody-mediated down-modulation of BTLA on lymphoid and myeloid cells of C57BL/6 and BALB/C BTLA allelic variants. Immunobiology 2010; 215: 570-578.

22 Ware CF, Sedy JR. TNF superfamily networks: bidirectional and interference pathways of the herpesvirus entry mediator (TNFSF14). Curr Opin Immunol 2011; 23: 627-631.

23 Watanabe N, Gavrieli M, Sedy JR, Yang J, Fallarino F, Loftin SK et al. BTLA is a lymphocyte inhibitory receptor with similarities to CTLA-4 and PD-1. Nat Immunol 2003; 4: 670-679.

24 Iwata A, Watanabe N, Oya Y, Owada T, Ikeda K, Suto A et al. Protective roles of $B$ and $T$ lymphocyte attenuator in NKT cell-mediated experimental hepatitis. J Immunol 2010; 184: 127-133.

25 Oya Y, Watanabe N, Owada T, Oki M, Hirose K, Suto A et al. Development of autoimmune hepatitis-like disease and production of autoantibodies to nuclear antigens in mice lacking $B$ and T lymphocyte attenuator. Arthritis Rheum 2008; 58: 2498-2510.

26 Del Rio ML, Pabst O, Ramirez P, Penuelas-Rivas G, Forster R, Rodriguez-Barbosa JI. The thymus is required for the ability of FTY720 to prolong skin allograft survival across different histocompatibility MHC barriers. Transpl Int 2007; 20: 895-903.

27 Del Rio ML, Kurtz J, Perez-Martinez C, Ghosh A, Perez-Simon JA, Rodriguez-Barbosa JI. B- and T-lymphocyte attenuator targeting protects against the acute phase of graft versus host reaction by inhibiting donor anti-host cytotoxicity. Transplantation 2011; 92 1085-1093.

28 Foy TM, Laman JD, Ledbetter JA, Aruffo A, Claassen E, Noelle RJ. gp39CD40 interactions are essential for germinal center formation and the development of B cell memory. J Exp Med 1994; 180: 157-163.

29 Monk NJ, Hargreaves RE, Marsh JE, Farrar CA, Sacks SH, Millrain M et al. Fc-dependent depletion of activated $\mathrm{T}$ cells occurs through CD4OL-specific antibody rather than costimulation blockade. Nat Med 2003; 9: 1275-1280.

30 Hargreaves RE, Monk NJ, Jurcevic S. Selective depletion of activated T cells: the CD4OL-specific antibody experience. Trends Mol Med 2004; 10: 130-135.

31 Unkeless JC. Characterization of a monoclonal antibody directed against mouse macrophage and lymphocyte Fc receptors. J Exp Med 1979; 150: 580-596.

32 Iwakoshi NN, Markees TG, Turgeon N, Thornley T, Cuthbert A, Leif J et al. Skin allograft maintenance in a new synchimeric model system of tolerance. J Immunol 2001; 167: 6623-6630.

33 Pearson T, Markees TG, Wicker LS, Serreze DV, Peterson LB, Mordes JP et al. NOD congenic mice genetically protected from autoimmune diabetes remain resistant to transplantation tolerance induction. Diabetes 2003; 52: 321-326.

34 Durie FH, Foy TM, Masters SR, Laman JD, Noelle RJ. The role of CD40 in the regulation of humoral and cell-mediated immunity. Immunol Today 1994; 15: 406-411.

35 Kashiwakuma D, Suto A, Hiramatsu Y, Ikeda K, Takatori H, Suzuki K et al. B and T lymphocyte attenuator suppresses IL-21 production from follicular Th cells and subsequent humoral immune responses. $\mathrm{J}$ Immunol 2010; 185: 2730-2736.

36 Hurchla MA, Sedy JR, Gavrieli M, Drake CG, Murphy TL, Murphy KM. $B$ and $T$ lymphocyte attenuator exhibits structural and expression polymorphisms and is highly Induced in anergic CD4+ T cells. $\mathrm{J}$ Immunol 2005; 174: 3377-3385.

37 Croft M, Benedict CA, Ware CF. Clinical targeting of the TNF and TNFR superfamilies. Nat Rev Drug Discov 2013; 12: 147-168.

38 Wekerle T, Grinyo JM. Belatacept: from rational design to clinical application. Transpl Int 2012; 25: 139-150.

39 Kim EJ, Kwun J, Gibby AC, Hong JJ, Farris AB, 3rd, Iwakoshi NN et al. Costimulation blockade alters germinal center responses and prevents antibody-mediated rejection. Am J Transp/ 2014; 14: 59-69.

40 Xie JH, Yamniuk AP, Borowski V, Kuhn R, Susulic V, Rex-Rabe S et al. Engineering of a novel anti-CD4OL domain antibody for treatment of autoimmune diseases. J Immunol 2014; 192: 4083-4092.

41 Chemnitz JM, Lanfranco AR, Braunstein I, Riley JL. B and $T$ lymphocyte attenuator-mediated signal transduction provides a potent inhibitory signal to primary human CD4 T cells that can be initiated by multiple phosphotyrosine motifs. J Immunol 2006; 176: 6603-6614.

42 Han P, Goularte OD, Rufner K, Wilkinson B, Kaye J. An inhibitory Ig superfamily protein expressed by lymphocytes and APCs is also an early marker of thymocyte positive selection. J Immunol 2004; 172: 5931-5939.

43 Sedy JR, Gavrieli M, Potter KG, Hurchla MA, Lindsley RC, Hildner K et al. $\mathrm{B}$ and $\mathrm{T}$ lymphocyte attenuator regulates $\mathrm{T}$ cell activation through interaction with herpesvirus entry mediator. Nat Immunol 2005; 6: 90-98.

44 Zeng $\mathrm{C}$, Wu T, Zhen Y, Xia XP, Zhao Y. BTLA, a new inhibitory B7 family receptor with a TNFR family ligand. Cell Mol Immunol2005; 2 427-432.

45 Cheung TC, Oborne LM, Steinberg MW, Macauley MG, Fukuyama S, Sanjo $\mathrm{H}$ et al. T cell intrinsic heterodimeric complexes between HVEM and BTLA determine receptivity to the surrounding microenvironment. J Immunol 2009; 183: 7286-7296.

46 Hurchla MA, Sedy JR, Murphy KM. Unexpected role of B and T lymphocyte attenuator in sustaining cell survival during chronic allostimulation. J Immunol 2007; 178: 6073-6082.

47 De Trez C, Schneider K, Potter K, Droin N, Fulton J, Norris PS et al. The inhibitory HVEM-BTLA pathway counter regulates lymphotoxin receptor signaling to achieve homeostasis of dendritic cells. J Immunol 2008; 180: 238-248.

48 Wang Y, Subudhi SK, Anders RA, Lo J, Sun Y, Blink S et al. The role of herpesvirus entry mediator as a negative regulator of $\mathrm{T}$ cell-mediated responses. J Clin Invest 2005; 115: 711-717.

49 Tao R, Wang L, Murphy KM, Fraser CC, Hancock WW. Regulatory T cell expression of herpesvirus entry mediator suppresses the function of $\mathrm{B}$ and T lymphocyte attenuator-positive effector T cells. J Immunol 2008; 180: 6649-6655.

50 Croft M. The TNF family in T cell differentiation and functionunanswered questions and future directions. Semin Immunol 2014; 26: 183-190.

51 Albring JC, Sandau MM, Rapaport AS, Edelson BT, Satpathy A, Mashayekhi $\mathrm{M}$ et al. Targeting of $\mathrm{B}$ and $\mathrm{T}$ lymphocyte associated (BTLA) prevents graft-versus-host disease without global immunosuppression. J Exp Med 2010; 207: 2551-2559.

52 Thangavelu G, Anderson CC. Divide and conquer: blocking graft versus host but not graft versus leukemia T cells with agonist BTLA co-inhibitory signals. Chimerism 2011; 2: 29-32.

53 Uchiyama M, Jin X, Matsuda H, Bashuda H, Imazuru T, Shimokawa T et al. An agonistic anti-BTLA mAb (3C10) induced generation of 
IL-10-dependent regulatory CD4 + T cells and prolongation of murine cardiac allograft. Transplantation 2014; 97: 301-309.

54 Alegre ML, Fu YX. Attenuation by targeting the B-and T-cell attenuator. Transplantation 2011; 92: 1075-1076.
55 Simpson TR, Li F, Montalvo-Ortiz W, Sepulveda MA, Bergerhoff K, Arce $\mathrm{F}$ et al. FC-dependent depletion of tumor-infiltrating regulatory $T$ cells co-defines the efficacy of anti-CTLA-4 therapy against melanoma. J Exp Med 2013; 210: 1695-1710. 